\title{
Brachytherapy: time to up the dose?
}

C urrent recommended doses of brachytherapy are insufficient to elicit the optimal response from many men who receive this treatment for prostatic malignancy. "Upwards of 50\% of patients being treated with brachytherapy in the USA may be receiving too low a dose of radiation to cure their cancers" states Nelson Stone, lead author of a new paper published in the Journal of Urology.

Brachytherapy has become a popular means of managing localized prostate cancer. Patients value the low impact of the procedure on potency and continence relative to alternatives such as hormone therapy and extirpative surgery. Rapid postprocedure resumption of everyday activities is another advantage. Short-term data have indicated that brachytherapy is associated with effective disease control. However, emerging longterm data show that a large proportion of patients are shortchanged at current recommended doses.

Stone and colleagues analyzed information collected over a 17 -year period at their institution, the Mount Sinai School of Medicine in New York. ${ }^{125}$ I seeds to a prescription dose of $160 \mathrm{~Gy}$ were implanted in just under 1,000 men with low-risk disease (defined as PSA $<10 \mathrm{ng} / \mathrm{ml}$, Gleason score $\leq 6$, stage $\leq \mathrm{T} 2 \mathrm{a}$,

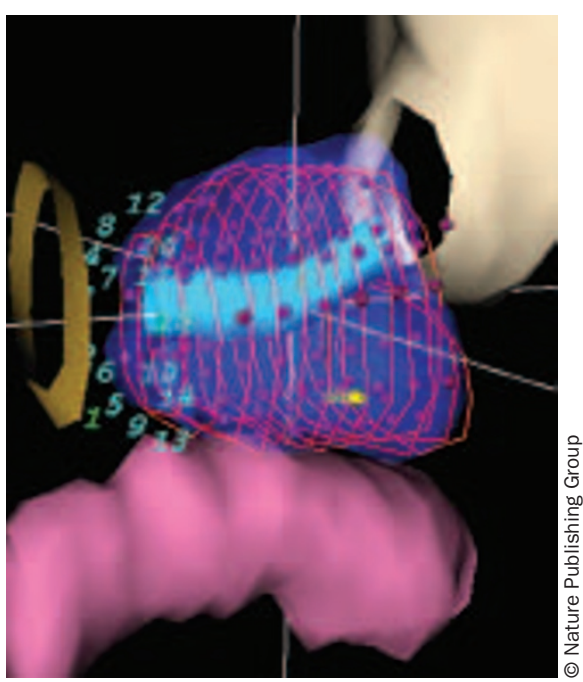

prostate volume $<50 \mathrm{ml}$ ). Men at intermediate and high risk of progression ( $n=499$ and 648, respectively) treated with a combination of brachytherapy and external beam radiation therapy (EBRT) were also included in the analysis.

At 12 years, the proportion of men who had experienced biochemical failure (PSA nadir plus $2 \mathrm{ng} / \mathrm{ml}$ ) was $12 \%, 21 \%$ and $33 \%$ in the low-risk, intermediaterisk and high-risk groups, respectively. The researchers used dosimetry data collected within 30 days of implantation to determine the biologically effective dose (BED) delivered to each patient. BED was significantly and independently associated with freedom from PSA relapse in all three groups. It was the only significant predictor of biopsy result at 2 years.

Stratification of outcomes data on the basis of BED - up to $150 \mathrm{~Gy} 2$, between 150 and 200 Gy2, or more than 200 Gy2 - revealed that markedly more men remained failure-free if treated with at least $200 \mathrm{~Gy} 2$, regardless of disease stage. The improvement was most dramatic $-14 \%$-in the intermediate-risk group.

"The current [prescription] dose recommendation for the most popular isotope, ${ }^{125} \mathrm{I}$, is $145 \mathrm{~Gy}$ ", explains Stone. "We have found that patients should receive 189 Gy to effectively control their cancer. That is a dose that is $30 \%$ higher!"

The 145 Gy dose to which Stone refers is recommended by the American Brachytherapy Society. Newly revised guidelines issued by the American College of Radiology and the American Society of Treating Radiation Oncologists suggest a dose in the 145-160 Gy range.

How is dosage escalation likely to impact the quality of life of patients? Unfortunately, Stone et al. did not report on morbidity relative to BED. Another research team has provided some insight into long-term adverse effects associated with brachytherapy, albeit only at a dose of 144 Gy (BED 170 Gy).

Michael Zelefsky and colleagues from the Memorial Sloan-Kettering Cancer
Center in New York studied a 448-strong cohort of men with low-risk prostate cancer (PSA $<10 \mathrm{ng} / \mathrm{ml}$, Gleason score $\leq 6$, stage $\leq$ T2a) who received ${ }^{125} \mathrm{I}$ implants. Relative to a smaller group of patients who were treated with 81 Gy intensitymodulated EBRT, the brachytherapy group had superior 7-year biochemical relapse-free survival (95\% versus 89\%).

The improved disease control afforded by brachytherapy was offset by greater toxicity. Almost $16 \%$ of men in the brachytherapy group suffered late grade 2 genitourinary events, compared with $4 \%$ in the EBRT group. Late grade 2 gastrointestinal toxicity data followed the same trend, albeit with lower incidences (5\% versus 1\%). Grade 3 events occurred very infrequently in both arms of the study.

Extrapolating from these findings, it is likely that aiming for a brachytherapy dose of 189 Gy - as recommended by Stone-will increase the likelihood of adverse events. Zelefsky et al. conclude their paper, published in Urology, by stating that the "apparent biochemical control advantage for brachytherapy might need to be considered in the presence of comorbidities or factors that could predict greater potential toxicities".

Stone's group are working towards identifying such "factors" in the hope that a personalized medicine approach will facilitate early identification of patients most likely to suffer serious radiationinduced adverse events. Having already discovered a number of relevant genetic mutations, the team is working towards development of a predictive blood test.

Suzanne J. Farley

Original articles Stone, N. N. et al. Influence of pretreatment and treatment factors on intermediate to long-term outcome after prostate brachytherapy. J. Urol. 185, 495-500 (2011) | Zelefsky, M. J. et al. Comparison of tumor control and toxicity outcomes of high-dose intensitymodulated radiotherapy and brachytherapy for patients with favorable risk prostate cancer. Urology doi:10.1016/ j.urology.2010.07.539 